\title{
Simulation and Design of University Area Network Scenario (UANS) using Cisco Packet Tracer
}

By Md. Anwar Hossain \& Mahabuba Zannat

Pabna University of Science and Technology

Abstract- Computer network has become the most significant issue in our day to day life. Networking companies depend on the proper functioning and analysis of their networks for education, administration, communication, e-library, automation, etc. Mainly interfacing with the network is induced by one of the other user/users to share some data with them. So, this paper is about communication among users present at remote sites, sharing this same network UANS. UANS stands for the University Area Network Scenario. So in this work the network is designed using Cisco Packet Tracer. The paper describes how the tool can be used to develop a simulation model of the Pabna University of Science and Technology, Pabna, Bangladesh. The study provides into various concepts such as topology design, IP address configuration and how to send information in the form of packets in a single network and the use of virtual Local Area Network (VLANs) to separate the traffic generated by a different department.

Keywords: computer networks, IP addresses, ping test, Cisco Packet Tracer Simulation Tool, VLANs, Router.

GJCST-G Classification: C.2.1

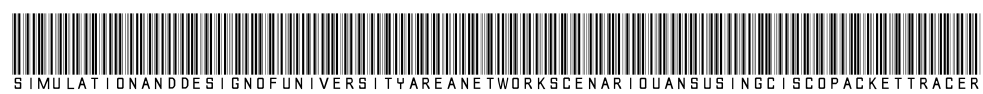

Strictly as per the compliance and regulations of:

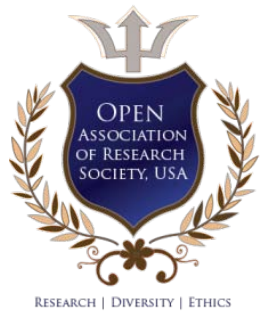

(C) 2019. Md. Anwar Hossain \& Mahabuba Zannat. This is a research/review paper, distributed under the terms of the Creative Commons Attribution-Noncommercial 3.0 Unported License http://creativecommons.org/licenses/by-nc/3.0/), permitting all noncommercial use, distribution, and reproduction in any medium, provided the original work is properly cited. 


\title{
Simulation and Design of University Area Network Scenario (UANS) using Cisco Packet Tracer
}

\author{
Md. Anwar Hossain ${ }^{\alpha} \&$ Mahabuba Zannat $^{\sigma}$
}

Abstract- Computer network has become the most significant issue in our day to day life. Networking companies depend on the proper functioning and analysis of their networks for education, administration, communication, e-library, automation, etc. Mainly interfacing with the network is induced by one of the other user/users to share some data with them. So, this paper is about communication among users present at remote sites, sharing this same network UANS. UANS stands for the University Area Network Scenario. So in this work the network is designed using Cisco Packet Tracer. The paper describes how the tool can be used to develop a simulation model of the Pabna University of Science and Technology, Pabna, Bangladesh. The study provides into various concepts such as topology design, IP address configuration and how to send information in the form of packets in a single network and the use of virtual Local Area Network (VLANs) to separate the traffic generated by a different department.

Keywords: computer networks, IP addresses, ping test, Cisco Packet Tracer Simulation Tool, VLANs, Router.

\section{INTRODUCTION}

$\longrightarrow$ omputer network borne out of the need to use personal computers for sharing information within an organization in the form of messages, sharing files, resources and so forth. Whether the organization is located in a small area like as in one building or spread over a large area, the need for networking the computers cannot be over emphasized. A university area network is an essential part of university system. A university network has several uses such as teaching, learning, result publishing, resource sharing, file sharing, communication, etc. The University Area Network design is about designing a topology of a network that is a LAN (Local Area Network) for a university in which various computers of different departments and buildings are set up so that they can interact and communicate with each other by interchanging data. To design a network

Author a: Assistant Professor, Department of Information and Communication Engineering, Faculty of Engineering andTechnology, Pabna University of Science and Technology, Pabna, Bangladesh. e-mail:manwar.ice@gmail.com

Author $\sigma$ : Student, Department of Information and Communication Engineering, Faculty of Engineering and Technology, Pabna University of Science and Technology, Pabna, Bangladesh.

e-mail: zannatbadhon95@gmail.com for a University which connects various departments and buildings to each other's, it puts forward communication among them. One of the purpose of networking is to reduce isolated users. The systems should be capable of communicating with others and should provide the desired information. A simulation tool offers a way to predict the impact on the network of a hardware upgrade, a change in topology. So in this dissertation, a UANS network is designed using Cisco Packet Tracer. Cisco Packet Tracer (CPT) is multitasking network simulation software that can be used to perform and analyze various network activities such as the implementation of different topologies, selection of optimum path based on various routers, analysis of different network configurations [4]. This University Area Network Scenario is about designing a topology of a network that is Local Area Network (LAN) for a university area in which different department has some computers in different buildings set up their network so that they can interact and communicate with each other by interchanging data.

\section{il. Technology to be Used}

CCNA: Cisco discovered Cisco Certified Network Associate (CCNA) to identify and analyzethe basic capability of the installation and maintenance of different size networks. This technology used for connecting various devices like routers, switches with each other for transferring and interchanging data. To contract a disciplined and trustworthy network is scalable too. Portability is one of the characteristics of this network application of the UANS. 
III. Basic Architecture

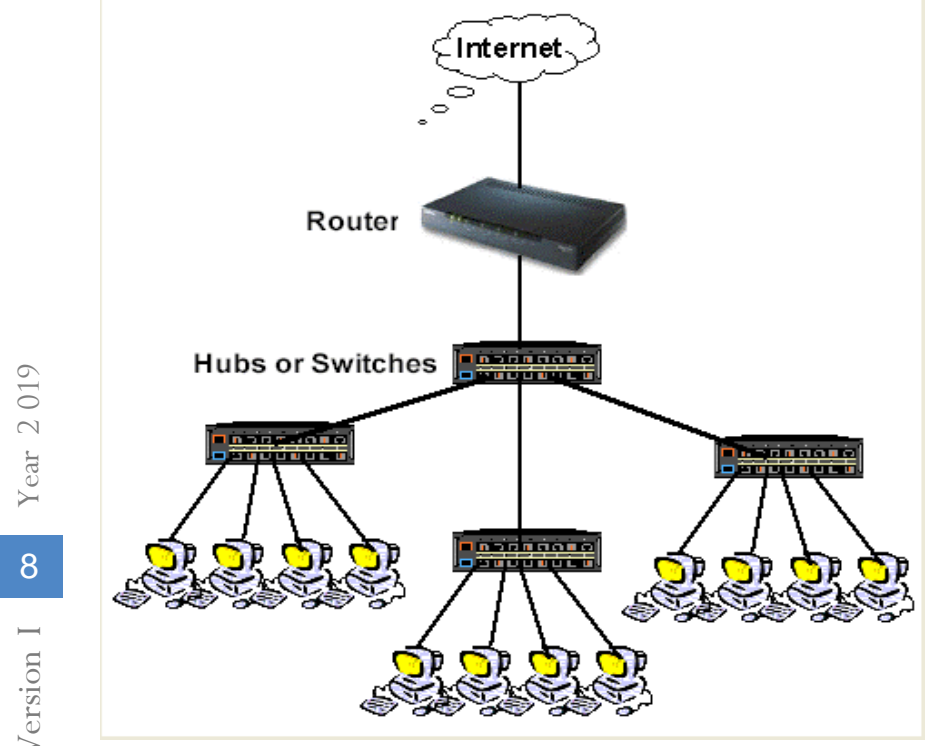

Fig. 1: The architecture of UANS use of various computers, routers, switches, VLAN connections, etc

\section{WORKING OF UANS}

This University Area Networking Scenario is to provide a well-connected, secure, reliable, dependable communication among different departments. Several departments can simply split the required data without any problem and can exchange their data without going physically to them thus conserving time and energy.

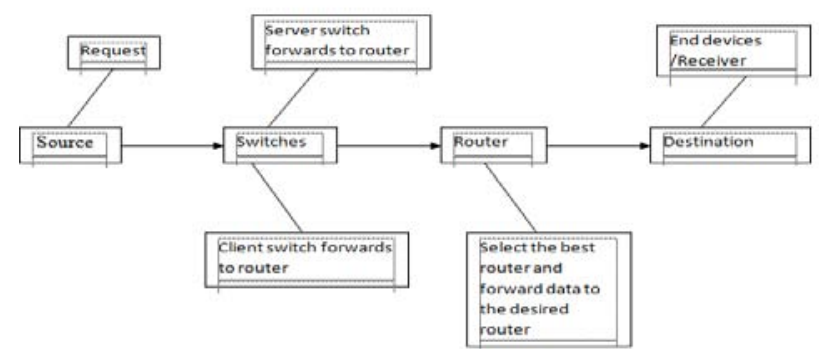

Fig. 2: A major component and their communication

\section{Requirements}

The following section discusses the requirements related to the interfaces used to communicate with lots of data. These data combine clients, software interfaces and other hardware that permit the system to carry out its task.

a) User Interface

The requirements represent in this module describes the UANS interface. The requirements are picked up to the main characteristics provided by the system. The necessities always maintain the activities interconnected with the subheading characteristics. b) Interface Formats

1. This screen is displayed the virtual topology designed for the university.

2. The network simulation, i.e. Cisco Packet Tracer (CPT) is simple, easy for implementation and straightforward.

3. It cultivates visual graphical user interface.

4. It will display twelve switches, different VLANs and one router interconnected with each other.

5. The Command Line Interface (CLI) use for configuration.

6. The CPT allows the user to run the project on run time mode or simulation.

7. When the administrator clicks on any device, then the graphical user interface is open for the particular device.

c) System Interface

In this UANS, giving data to end device, a Graphical User Interface is needed and appear a login form to the authorized user. We can reset the password by the help of network administration. Whereas unauthorized user prohibits to access the network.

d) Hardware Interface

To run the simulator software (i.e. CPT) some basic hardware requirements are need. That are-

- RAM:512MB

- CPU: Intel Pentium Dual core

- Storage:500MB of free disk space

- Display Resolution:800*600

- Adobe Flash Player.

Recommended Hardware:

- RAM: > $512 \mathrm{MB}$

- $\quad$ CPU: Intel Pentium III $1.0 \mathrm{GHz}$

- Display resolution: $1024 \times 768$

- Storage: $300 \mathrm{MB}$ free disk space

For run the module, the hardware requirements are:

- 12 Switches (Cisco 2950 switch)

- 1 Router (Cisco 1841 router)

- Computer Systems (Generic)

- One Computer system for server

- Cross over cable

- Straight through cable

e) Software Interfaces

The requirements required in the UANS are as follows:

- $\quad$ Operating System: Microsoft Windows 7.

- Adobe Flash Player.

- Cisco Packet Tracer

f) Communication Interfaces

- The system will execute over the university area network.

- The system based on a client-server application where the server provide data services only. 


\section{IP ADDRESSING}

An Internet Protocol address is a logical, numerical address that is assigned to every single computer, printer, switch, router or any device that is part of an IP based network [5]. No network architecture exists without an IP address. Due to an IP address is logical address, so it used to identify the specific nodes in the network. So the IP address gives the location an address so that they can interact with each other via email or so on. An IP address is a 32-bit number. It has two versions one is IPv4 and other is IPv6. There are five classes of IPv4 address shown in Table-1.

Table 1: Classes of IP addresses

\begin{tabular}{|c|c|}
\hline ADDRESS CLASS & VALUE IN $1^{\text {ST }}$ OCTET \\
\hline A & $1-126$ \\
\hline$B$ & $128-191$ \\
\hline C & $192-223$ \\
\hline$D$ & $224-239$ \\
\hline E & $240-255$ \\
\hline
\end{tabular}

In the UANS is used IPv4. An IPv4 is of 32 bits numbers. The address is written in dotted-decimal notation represent it X.X.X.X each octet divide by a dot. For UANS network the IP Address is used 192.168.0.0 with a subnet mask of 255.255.0.0. IP 192.168.0.0 is the class $\mathrm{C}$ address. IP address divide among various VLANs and ports for communication. The greater IP address is divide into small parts by using VLSM (Various Length Subnet Mask). VLSM is a method that categorized an IP address into a subnet and shares it according to the necessity of a network.

\section{SCOPE OF UANS}

This project gives us an effective method to connect among all computers in the respective university area. Apart from interconnection, this topology has been created keeping in mind the matter of cost. One of the important points is security and authentication to oppose unauthorized access. This topology transmits data very fast from one place to another.

\section{Vili. Result of UANS}

In Fig.3 show the complete diagram of University Area Network and it consists of router, switches and end devices. Here the different department's personal computers are connected with switch. IP address and subnet mask are assigned to all the departments and area of this UANS.

IP Address of personal computer (PC):

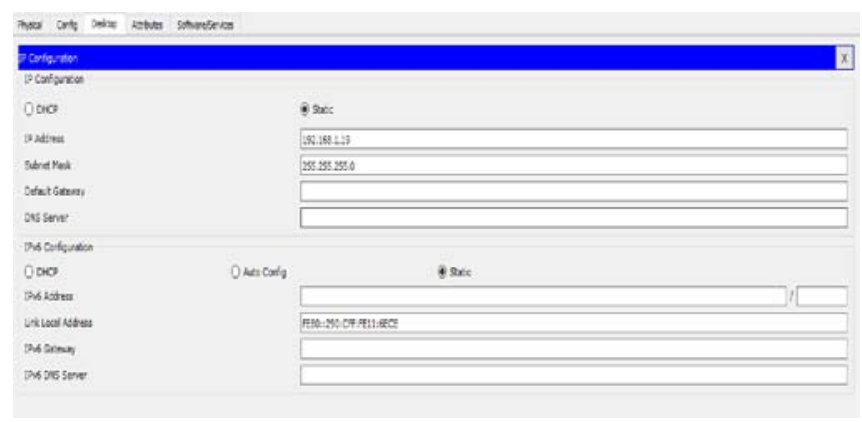

Fig. 4: IP Address accessing for PC-0

Table 2 shows the IP Addresses all PCs which are used in UANS network.

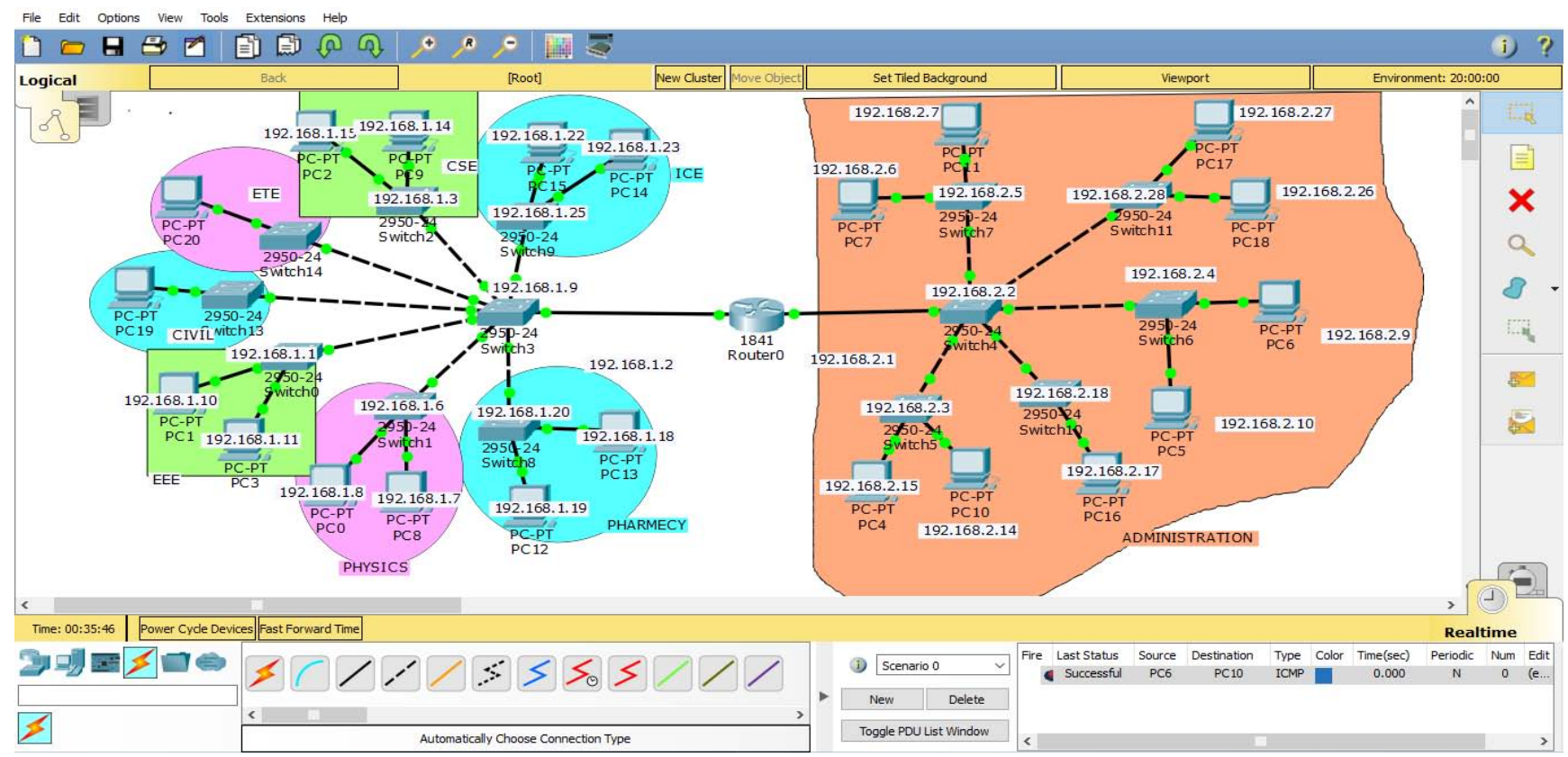

Fig. 3: The complete diagram of University Area Network Scenario created in Packet Tracer environment 
Table 2: List of IP Address of PCs

\begin{tabular}{|c|c|}
\hline PC & IPADDRESS \\
\hline PC-0 & 192.168 .1 .8 \\
\hline PC-1 & 192.168 .1 .10 \\
\hline PC-2 & 192.168 .1 .15 \\
\hline PC-3 & 192.168.1.11 \\
\hline PC-4 & 192.168.2.15 \\
\hline PC-5 & 192.168 .2 .10 \\
\hline PC-6 & 192.168 .2 .9 \\
\hline PC-7 & 192.168.2.6 \\
\hline PC-8 & 192.168 .1 .7 \\
\hline PC-9 & 192.168 .1 .14 \\
\hline PC-10 & 192.1682 .14 \\
\hline PC-11 & 192.168 .2 .7 \\
\hline $\mathrm{PC}-12$ & 192.168.1.19 \\
\hline $\mathrm{PC}-13$ & 192.168 .1 .18 \\
\hline PC-14 & 192.168.1.23 \\
\hline $\mathrm{PC}-15$ & 192.168 .1 .22 \\
\hline $\mathrm{PC}-16$ & 192.168.2.17 \\
\hline PC-17 & 192.168.2.27 \\
\hline PC-18 & 192.168.2.26 \\
\hline PC-19 & 192.168.1.52 \\
\hline PC-20 & 192.168 .1 .53 \\
\hline
\end{tabular}

Command line interface (CLI) for Switch:

switch>enable

switch\#config t

Enter configuration commands. One per line. End with CNTL/Z. switch(config)\#hostname s1

switch(config)\#interface vlan1

switch(config-if)\#IP address 192.168.1.1 255.255.0.0

$\operatorname{switch}$ (config-if)\#no shut

\section{Fig. 4: Configuration of a switch}

Table 3 shows the IP Addresses all switches which are used in the UANS network.

Table 3: List of IP Address of switches

\begin{tabular}{|c|c|}
\hline SWITCH & IP ADDRESS \\
\hline SWITCH-0 & 192.168 .1 .1 \\
\hline SWITCH-1 & 192.168 .1 .6 \\
\hline SWITCH-2 & 192.168 .1 .3 \\
\hline SWITCH-3 & 192.168 .1 .9 \\
\hline SWITCH-4 & 192.168 .2 .2 \\
\hline SWITCH-5 & 192.168 .2 .3 \\
\hline SWITCH-6 & 192.168 .2 .4 \\
\hline SWITCH-7 & 192.168 .2 .5 \\
\hline SWITCH-8 & 192.168 .1 .20 \\
\hline SWITCH-9 & 192.168 .1 .25 \\
\hline SWITCH-10 & 192.168 .2 .18 \\
\hline SWITCH-11 & 192.168 .2 .28 \\
\hline SWITCH-13 & 192.168 .1 .51 \\
\hline SWITCH-14 & 192.168 .1 .50 \\
\hline
\end{tabular}

Command Line Interface (CLI) for Router:

Continue with configuration dialog? (yes/no)

no

Press RETURN to get started!

Router >enable|

Router\#config t

Enter configuration commands, one per line. End with CNTL/Z.

Router(config)\#interface fa

Router(config)\#interface fastEtharnet $0 / 0$

Router(config-if)\#ip address192.168.1.2 255.255.255.0

Router(config-if)\#no shut

\%LINK-5-CHANGED: Interface FastEthernet $0 / \theta$, changed state to up

\%LINK-5-UPDOWN: Line protocol on Interface FastEthernet $\theta / \theta$, changed state to up

Router(config-if)\#exit

Router(config)\#interface fastEtharnet $1 / 0$

Router(config-if)\#ip address192.168.2.1 255.255.255.0

Router(config-if)\#no shut

\%LINK-5-CHANGED: Interface FastEthernet $0 / 0$, changed state to up

\%LINK-5-UPDOWN: Line protocol on Interface FastEthernet $0 / 0$, changed state to up

\section{Fig. 5: Configuration of router}

8.1 Ping Test: Network connectivity and communication can be tested using ping command, followed by the domain name or the IP address of the device (equipment) one wishes to verify connectivity. Two VLANs have been added to the existing network and the ping command was performed to verify if the devices connected to those VLANs are communicating with the rest of the nodes on the network. [2]

Academic area $\longrightarrow$ Administration area
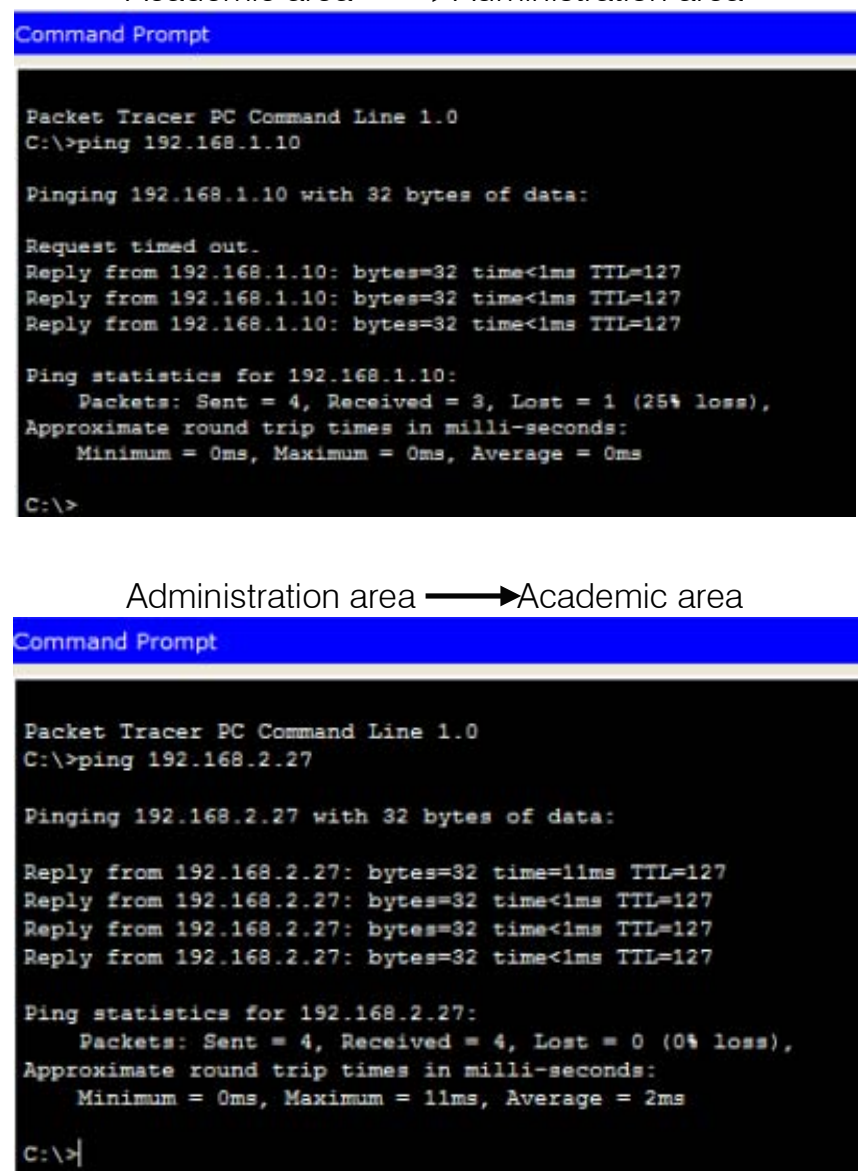


\section{Civil $\rightarrow$ ICE}

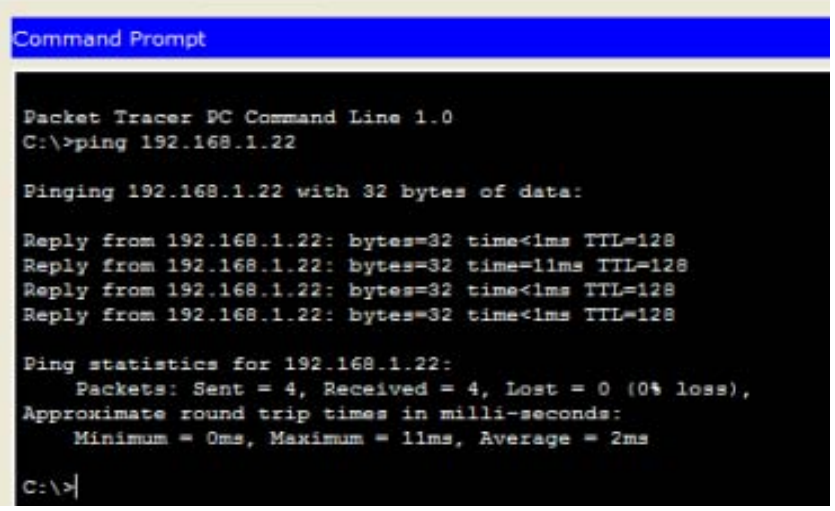

Data transfer one pc to another pc

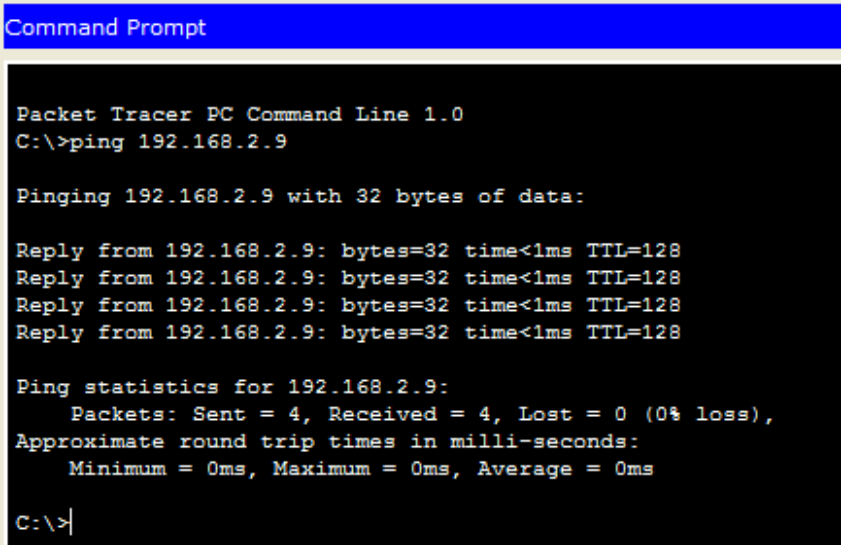

Fig. 5: Checking result using 'ping' command

\section{Conclusion}

In this paper, a UANS (University Area Network Scenario) that uses networking topology implemented with concepts like VLANs in a multiple area networks using Cisco Packet Tracer. VLANs have been used to logical UANS and with the aid of a router and switch configurations, data packet routed from one device to another. The procedures provide a design and approach for UANS using VLANs for an end to end IP network connectivity for next generation network architecture implementations. To design the network for UANS produces the substructure for all the service framework such as security of the network, wireless area network, operational efficiencies, virtual learning environments, and secure classrooms. Finally, the network foundation services such as switching, routing multicast, and high availability are giving for the UNAS.

\section{Future Work}

The configuration and specifications are for the initial prototype and can further be developed and additional functionality can be added to increase support and coverage.

\section{References Références Referencias}

1. www.wikipedia.com

2. Nathaniel S. Tarkaa, Paul I. Iannah, Isaac T. Iber, "Design and Simulation of Local Area Network Using Cisco Packet Tracer", The International Journal of Engineering and Science (IJES), vol.6, pp 63-73, 2017

3. Paulami Pathak, SayantiMajumder, Chandra Mondal, Prof. Manikandan K, "College Network Scenario Implementation by using Cisco Packet Tracer", International Journal of Advanced Research in Computer and Communication Engineering, Vol. 7, January 2018.

4. Garima Jain, NasreenNoorani, NishaKiran, Sourabh Sharma, "Designing \& simulation of topology network using Packet Tracer", International Research Journal of Engineering and Technology (IRJET), 2(2), 2015.

5. www.techopedia.com

6. Jitender Singh, Anshu Rani, "Implementation of College Network Scenario Module by Using CCNA", International Journal of Recent Development in Engineering and Technology, Vol. 3, July 2014.

7. Cisco Certified Network Associate Study Guide fifth edition by Todd Lammle

8. http://www.ciscopress.com/articles/article.asp? $p=3$ 28773\&seqNum $=3$

9. Interconnecting Cisco Devices Part 1 by Cisco

10. Interconnecting Cisco Devices Part 2 by Cisco

11. www.wikipedia.org/wiki/computer_network

12. www.wikipedia.org/wiki/local_area_network 